\title{
Padrões e tendências a médio prazo da contaminação por resíduos sólidos na praia de Boa Viagem, Nordeste do Brasil
}

\section{Medium-term patterns and trends of solid waste contamination on the beach of Boa Viagem, Northeast Brazil}

\author{
Jacqueline Santos Silva-Cavalcanti ${ }^{\text {ad }}$, Maria Christina Barbosa Araujo ${ }^{\text {be }}$, Monica Ferreira \\ Costa $^{\text {cf }}$
}

${ }^{\mathrm{a}}$ Universidade Federal Rural de Pernambuco, ${ }^{\mathrm{b}}$ Universidade Federal do Rio Grande do Norte, ${ }^{\mathrm{c}}$ Universidade Federal de Pernambuco

d jacqueline.silva@pq.cnpq.br, ${ }^{\mathrm{e}}$ chrisaraujo1@hotmail.com, ${ }^{\mathrm{f}} \mathrm{mfc} @$ ufpe.br

\begin{abstract}
RESUMO
A quantidade, diversidade e composição do lixo encontrado ao longo de uma praia arenosa do nordeste do Brasil (praia de Boa Viagem) foram descritos neste trabalho. Variações temporais e espaciais da contaminação por lixo marinho depositados na linha do deixa num período de três anos foram examinadas. As amostras foram coletadas durante as estações seca (janeiro, fevereiro, março) e chuvosa (junho, julho, agosto) de 2005 a 2007. A praia foi amostrada uma vez por mês para contagem e classificação de todos os itens visíveis dentro de um transecto com 1m de largura centrado na linha do deixa. Foram encontradas diferenças significativas na diversidade e quantidade de lixo marinho encontrado ao longo das duas estações do ano. Um total de 165.882 itens foram depositados na praia de Boa Viagem. A categoria plástico representou 74,3\% dos resíduos sólidos encontrados na praia de Boa Viagem. A quantidade de plástico foi significativamente maior $(\mathrm{p}<0,05)$ do que a matéria orgânica e madeira. Diferenças significativas $(\mathrm{p}<0,05)$ foram observadas na diversidade de lixo durante eventos de El Niño. Estas análises destacam os problemas que devem ser abordados na gestão futura de áreas de praia.
\end{abstract}

Palavras-chave: lixo marinho; padrão de contaminação; praias tropicais; linha do deixa.

\begin{abstract}
The quantity, diversity and composition of solid waste found along a developed coastline in Northeast Brazil are described. Small to medium-scale temporal and spatial variations in marine debris deposited over strandline in a period of three years were examined. Sampling was conducted during the dry (January, February, March) and rainy (June, July, August) seasons of 2005 to 2007. Samples were collected once a month for counting and sorting all visible items within a $1 \mathrm{~m}$ wide belt transect centered at the strandline. The very small plastic fragments $(<1 \mathrm{~cm})$ were not recorded in the sample because it was very difficult to spot and quantify them in a reliable manner during this sort of survey. Significant differences were found in diversity and quantity of marine debris along two seasons at Boa Viagem beach. Large numbers of solid waste items were counted in each year along three years. A total amount of 165.882 items was counted at the beach of Boa Viagem. The plastic category was the most important type of solid waste found at Boa Viagem. This category represented $74,3 \%$ of all solid waste found at Boa Viagem beach. Plastic waste was significantly greater $(p<0,05)$ than organic matter and wood. Significant differences $(p<0,05)$ were observed in marine debris diversity along El-Niño events. These analyses highlight problems that should be addressed in future management of sandy beaches.
\end{abstract}

Keywords: marine debris; contamination patterns; tropical sandy beaches; strandline.

\section{Introdução}

O monitoramento ambiental é essencial para subsidiar um padrão mínimo de qualidade e conservação das praias, aumentando a satisfação dos seus usuários, bem como da população local (Silva et al. 2008). A qualidade de uma praia arenosa é principalmente percebida e/ou avaliada através da limpeza da areia e da qualidade bacteriológica da água (Silva et al. 2008, Ballance et al. 2000, Dias-Filho et al. 2011a). Métodos rápidos e confiáveis para diagnóstico da contaminação de praias por resíduos sólidos são descritos como sendo uma importante ferramenta de avaliação do status deste tipo de contaminação (Ribic 1998, Araújo \& Costa 2006a, Araújo \& Costa 2006b, Silva-Cavalcanti et al. 2009, Soares et al. 2007). Um desses métodos de monitoramento consiste na contagem de itens encontrados ao longo da linha do deixa (linha mais alta da maré). 
A maioria das pesquisas sobre a contaminação por resíduos sólidos ao redor do mundo se limita a um diagnóstico pontual (no tempo e/ou espaço) da contaminação em praias. Trabalhos baseados em séries temporais são raros no Brasil (Santos et al. 2005, Tourinho et al. 2007, Portz et al. 2011, Tourinho \& Filmann 2011, Vieira et al. 2011) e escassos no resto do mundo (Walker et al. 1997, Ribic 1998, Hess et al. 1999, Galgani et al. 2000, Józwiak 2005, Morishige et al. 2007). O valor de dados contínuos do monitoramento da contaminação por resíduos sólidos está em estabelecer tendências temporais do aumento ou diminuição da qualidade da praia e consequentemente da qualidade recreacional para os usuários. Estas informações podem ainda refletir que diferentes tipos de itens e variáveis ambientais influenciam a tendência geral de acumulação destes resíduos na praia.

A praia de Boa Viagem é uma praia arenosa urbana, de grande importância turística no estado de Pernambuco (figura 1). Dentro da classificação de Smith (1991) baseada na dinâmica de urbanização das praias, a praia de Boa Viagem pode ser considerada uma 'cidade resort'. Esta praia é uma das poucas oportunidades que os habitantes do Recife e sua Região Metropolitana têm de ter contato com o ambiente marinho. Boa Viagem é intensamente utilizada no verão (Silva et al. 2006, 2008), e este uso intenso influencia sua qualidade ambiental. Mesmo contando com serviços públicos de varrição, esta praia apresenta altos níveis de contaminação por resíduos sólidos, sendo sua principal fonte de contaminação os próprios usuários (Silva-Cavalcanti et al. 2009).

A praia de Boa Viagem é considerada uma praia dissipativa. Geologicamente, a área é relativamente plana, com baixa declividade entre a pós-praia e a antepraia, e ambiente praial de larguras variáveis, preenchido por sedimentos quaternários arenosos com granulometria de fina à média (Rollnic 2008). De acordo com Gregório (2003), que analisou a variação volumétrica de sedimentos durante dois anos na praia de Boa Viagem, a praia apresenta pequena variação; ou seja, possui um maior equilíbrio no volume sedimentar, especialmente na parte mais central, e uma maior variação em direção aos extremos, especialmente ao sul da área.

Os principais rios que desembocam na área (figura 1) apresentam regime pluvial. Ao norte da área, o principal rio é o Capibaribe, com $253 \mathrm{~km}$ de extensão; área de drenagem de $7.438 \mathrm{~km}^{2}$ e vazão média anual de $20,05 \mathrm{~m}^{3} / \mathrm{s}$. Ao sul, os principais rios são o Jaboatão, com extensão aproximada de $75 \mathrm{~km}$, área de drenagem com 413,1 $\mathrm{km}^{2}$ e vazão média anual de $12,80 \mathrm{~m}^{3} / \mathrm{s}$; e o Pirapama com $80 \mathrm{~km}$ de extensão, área de drenagem com cerca de $600 \mathrm{~km}^{2}$ e vazão média anual de 9,41 $\mathrm{m}^{3} / \mathrm{s}$. Os rios Jaboatão e Pirapama desembocam em um mesmo ponto, formando o estuário de Barra de Jangadas (Leite 2009).

De acordo com a Agência Estadual de Meio Ambiente e Recursos Hídricos (CPRH), as bacias dos rios Jaboatão e Pirapama possuem indústrias que apresentam uma produção muito diversificada. Além da poluição dos cursos d'água por origem industrial, existe a poluição de origem doméstica, representada tanto por esgotos como por depósitos de lixo em áreas próximas das margens (Leite 2009).

A contaminação das águas do estuário do rio Capibaribe também é visivelmente crítica. A presença de inúmeras galerias de esgotos ao longo do rio, e principalmente no trecho mais próximo à foz, indica que a área recebe uma grande contribuição de águas residuárias industriais e domésticas no estuário. Além disto, a área se encontra impactada por grande quantidade de resíduos sólidos (Nóbrega 2011).

Segundo Rollnic (2008), a área litorânea na qual Boa Viagem se insere está sujeita a um alto potencial de transporte. Especialmente no período chuvoso, a drenagem do sistema Jaboatão-Pirapama se desloca até a praia de Boa Viagem devido à predominância dos ventos SE. A presença de recifes próximos à costa e a norte do estuário Jaboatão-Pirapama parece atuar como guia corrente natural, direcionando a pluma estuarina entre a linha de costa e o recife costeiro, influenciando diretamente a qualidade de suas águas e o aporte de sedimentos para a região.

Esta pesquisa teve como objetivo identificar um padrão de contaminação da praia da Boa Viagem por resíduos sólidos ao longo de três anos consecutivos (2005-2007) e possíveis diferenças na deposição entre anos e estações do ano. Através dos resultados gerados espera-se indicar onde e quando a praia tornou-se mais vulnerável a esta contaminação.

\section{Materiais e Métodos}

A trajetória de $8 \mathrm{~km}$ de praia foi percorrida 18 vezes (6x/ano) ao longo de três anos de coleta (2005 a 2007). Cada ano foi amostrado seis vezes, sendo três meses de verão (dezembro, janeiro e fevereiro) e três meses de inverno (junho, julho e agosto). As amostragens foram realizadas durante a maré baixa em ambos os períodos (verão e inverno). O período de verão se caracteriza por uma maior concentração de usuários na praia (Silva et al. 2008) e menor influência do rio Jaboatão na deposição de resíduos. A média de precipitação nessa época do ano para os anos amostrados foi de 63,5 Desvpad \pm 30,9 mm (www.inmet.gov.br). Já o período de inverno apresenta normalmente uma menor frequência de usuários (Silva et al. 2008) e uma maior influência do fluxo do rio Jaboatão sobre os resíduos depositados ao longo da faixa de praia (SilvaCavalcanti et al. 2009). A precipitação média mensal no inverno para os anos amostrados foi de 247,8 Desvpad \pm 117,5 mm (www.inmet.gov.br).

O trecho foi amostrado uma vez ao mês, em oportunidade escolhida de forma aleatória entre dias de semana e finais de semana, padronizando-se apenas a maré de sizígia com amplitude de 0,5m. Todos os objetos facilmente visíveis na linha do deixa (figura 1), considerando-se aproximadamente $1 \mathrm{~m}$ de largura (50 $\mathrm{cm}$ para cada lado a partir do centro) foram contados, 
classificados e registrados. Para a contagem, foi adotado o critério de identificação de itens com tamanho superior a $1 \mathrm{~cm}$, independentemente de sua composição. Os itens inferiores ao tamanho de identificação não foram considerados nesta amostragem.

As amostragens foram realizadas no período da manhã, antes do serviço de limpeza pública, que acontece duas vezes ao dia (Silva-Cavalcanti et al. 2009). Todos os resíduos contados foram enquadrados dentro de uma das seguintes categorias: plástico, papel, vidro, madeira, matéria orgânica, aço, alumínio e resto de material de construção. $\mathrm{O}$ item bituca de cigarro foi considerado como plástico por ser considerado o filtro como o composto mais persistente no ambiente. A análise de variância Two-way ANOVA foi utilizada para avaliar quais categorias eram diferentes estatisticamente. Além disso, para cada item de plástico foi considerada uma subcategoria de forma, material e uso (ex. tampas, garrafas PET, colheres, copos etc.). Os plásticos foram ainda subdivididos entre suas duas fontes mais prováveis: usuários da praia e aporte fluvial (Silva-Iñiguez \& Fischer 2003).

\subsection{Tratamento estatístico}

O índice de Shanon-Winner foi usado para medir a diversidade das amostras. Two-Way ANOVA foi realizada para verificar a existência de diferença significativa entre a diversidade média observada, número total de itens, diversidade do total de itens, número de itens de plástico e diversidade de itens de plástico nos anos amostrados. Onde a ANOVA mostrou-se significativa $(\mathrm{p}<0,05)$, o teste a posteriori Scheffé foi usado para determinar quais médias eram significativamente diferentes a um nível de probabilidade de 0,05 .

O teste $t$ foi realizado para testar a diferença entre as médias da diversidade nos dois períodos do ano amostrados (verão/inverno). As médias foram consideradas diferentes a um nível de significância de $95 \%$.

\section{Resultados}

\subsection{Composição e abundância dos resíduos sólidos na praia de Boa Viagem}

De 2005 a 2007 um total de 165.882 itens foi amostrado ao longo dos $8 \mathrm{~km}$ da praia, com uma deposição anual variando de um mínimo de 30.263 itens em 2005 a um máximo de 89.415 itens em 2006. O valor médio de deposição dos resíduos sólidos foi de 55.294 Desvpad \pm 30.606 itens/ano, nos três anos amostrados (Fig. 3).

A categoria plástico foi a mais abundante. Esta categoria representou 74,3\% (125.353 itens) de todo o lixo contado e foi significativamente maior $(\mathrm{p}<0,05)$ do que a matéria orgânica (10,3\%; 17.166 itens) e a madeira (9,9\%; 16.496 itens), que constituíram a segunda e terceira categorias de resíduos sólidos mais abundantes ( $\mathrm{p}>0,05)$, respectivamente. Apenas $1 \%$ foi a soma de todos os outros tipos de resíduos juntos (papel 0,81\%, vidro 0,12\% e alumínio 0,11\%).

Os itens plásticos mais abundantes de 2005 a 2007 foram a bituca de cigarro/filtro (24.803), embalagens de comida (11.451) e canudo (10.786).

Provavelmente a principal fonte de resíduos sólidos para a praia de Boa Viagem corresponde aos usuários da praia, já que aproximadamente $80 \%$ de todo o resíduo sólido encontrado na praia durante os três anos de estudo esteve relacionado ao usuário, como canudos, copos plásticos, etc. A segunda fonte, com cerca de $18 \%$ dos itens, foi a origem terrestre, pela deposição de itens que sofreram transporte através do estuário Barra de Jangada, situado a aproximadamente $16 \mathrm{~km}$ de distância do extremo norte da área. Apenas 2,8\% dos itens (redes, light stick e cordas) têm origem marinha, principalmente relacionada à atividade da pesca.

\subsection{Variação sazonal e interanual da diversidade de resíduos sólidos na praia de Boa Viagem}

A ANOVA demonstrou existir diferença significativa $(\mathrm{p}<0,05)$ na diversidade de resíduos sólidos entre os anos amostrados. A diversidade foi maior no ano de 2005 do que nos demais (2006 e 2007) (figura 2). Os testes de Scheffé a posteriori demonstraram que a diversidade no verão de 2005 (v1) foi significativamente maior $(\mathrm{p}<0,05)$ do que nos demais verões amostrados (v2-2006 e v3-2007) (tabela 1).

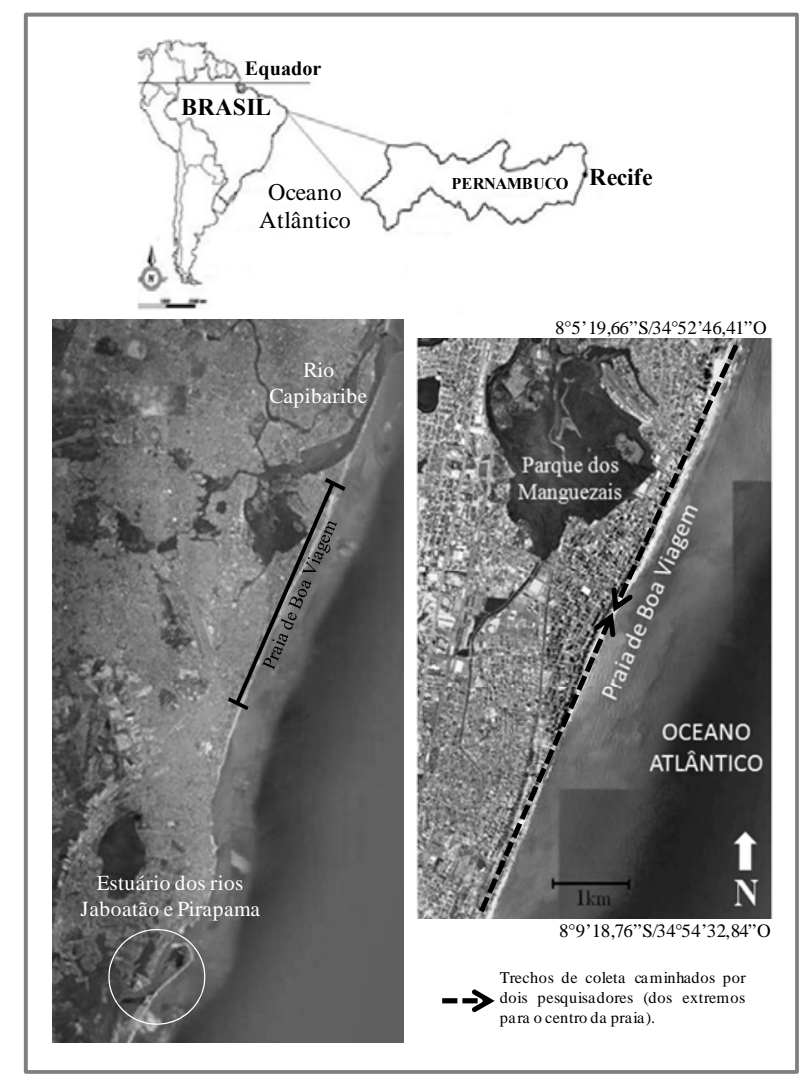

Figura 1: Localização da Praia de Boa Viagem, Recife, Pernambuco 


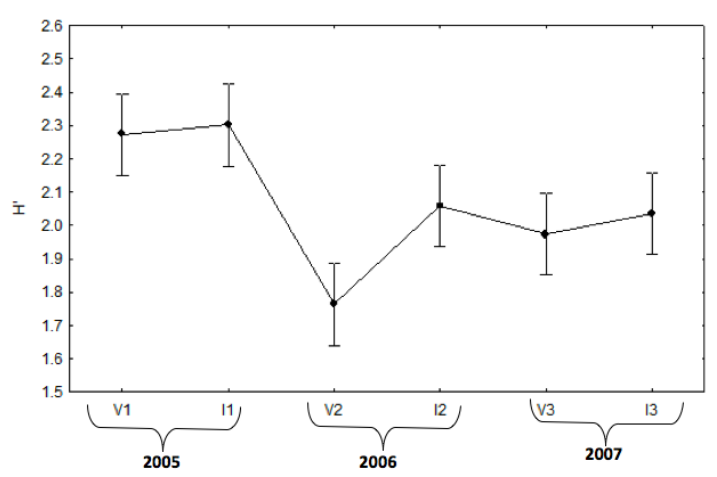

Figura 2: Índice de diversidade dos resíduos sólidos ao longo dos diferentes anos amostrados (2005-2007) e em diferentes estações do ano

Tabela 1: Resultado do teste a posteriori Scheffé para a diversidade de resíduos ao longo dos diferentes anos coletados (2005-2007)

\begin{tabular}{ccl}
\hline Ano & Período de Amostragem & \multicolumn{1}{c}{$\mathbf{p} \leq \mathbf{0 , 0 5}$} \\
\hline \multirow{2}{*}{2005} & V1 & I1 V2 I2 V3 I3 \\
& I1 & V1 V2 I2 V3 I3 \\
$\mathbf{2 0 0 6}$ & V2 & V1 I1 I2 V3 I3 \\
& I2 & V1 I1 V2 $\underline{\text { V3 I3 }}$ \\
$\mathbf{2 0 0 7}$ & V3 & V1 I1 $\underline{\text { V2 I2 I3 }}$ \\
\hline
\end{tabular}

\subsection{Diferença entre as diversidades médias em} diferentes estações do ano (verão $x$ inverno)

Em todos os anos amostrados, a diversidade (figura 2) e a quantidade de itens plásticos (figura 3) foram maiores no inverno do que no verão. A razão de acumulação de itens plásticos ao longo da linha do deixa na praia de Boa Viagem variou de 459 itens $/ \mathrm{km}$ no verão de 2005 a 626 itens/km durante o inverno de 2007. O maior número de itens plásticos amostrados mensalmente ocorreu nos meses de julho (5.811 Desvpad \pm 8.21 itens $/ \mathrm{m}$ linear) e fevereiro (4.193 Desvpad \pm 1.181 itens $/ \mathrm{m}$ linear).

$\mathrm{O}$ teste $\mathrm{t}$ mostrou que existe diferença significativa (p $<0,05)$ na diversidade média de plásticos no período amostrado. A diversidade de plásticos foi maior no inverno $\left(H^{\prime}=2,131\right.$ Desvpad $\left.\pm 0,21\right)$ do que no verão $\left(H^{\prime}=2,003\right.$ Desvpad $\left.\pm 0,37\right)$.

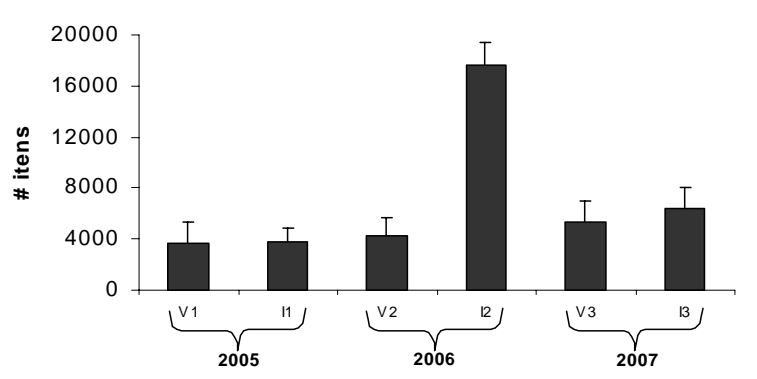

Figura 3: Quantidade de itens de resíduos sólidos coletados ao longo de diferentes estações do ano (verão e inverno) durante o período 2005-2007

\subsection{Variação temporal da quantidade de resíduos originados pelos usuários}

A análise de MDS demonstrou uma diferença de deposição de alguns itens gerados pelo usuário entre os meses do ano (figura 4). Os itens que apresentaram ocorrência mensal foram copo, sachet de bronzeador, frasquinho de água oxigenada, tampa, colher e canudo, sendo bastante expressivos na estação do verão austral (dezembro a fevereiro) (figura 4).

\subsection{Levantamento dos valores de chuva e velocidade do vento}

O padrão de precipitação para os anos amostrados mostrou-se maior nos meses de junho, julho e agosto. O maior valor de precipitação ocorreu no mês de junho de 2006 (709 mm). A precipitação nos anos amostrados não apresentou diferença significativa em relação à média histórica $(\mathrm{p}>0,05)$. Foram observados valores destoantes da velocidade do vento em relação à média histórica recente (2000-2007). Essa média recente para os meses de janeiro, fevereiro e março foi, respectivamente, de 2,4 Desvpad \pm 0,3, 2,3 Desvpad \pm 0,3 e 2,4 Desvpad $\pm 0,4 \mathrm{~m} . \mathrm{s}^{-1}$. Para o ano de 2005, os valores encontrados foram de 2,7, 2,5 e 2,4 m.s $\mathrm{s}^{-1}$ para janeiro, fevereiro e março, respectivamente. A velocidade do vento nos meses amostrados não apresentou diferenças significas $(p>0,05)$ em relação à média histórica recente. 


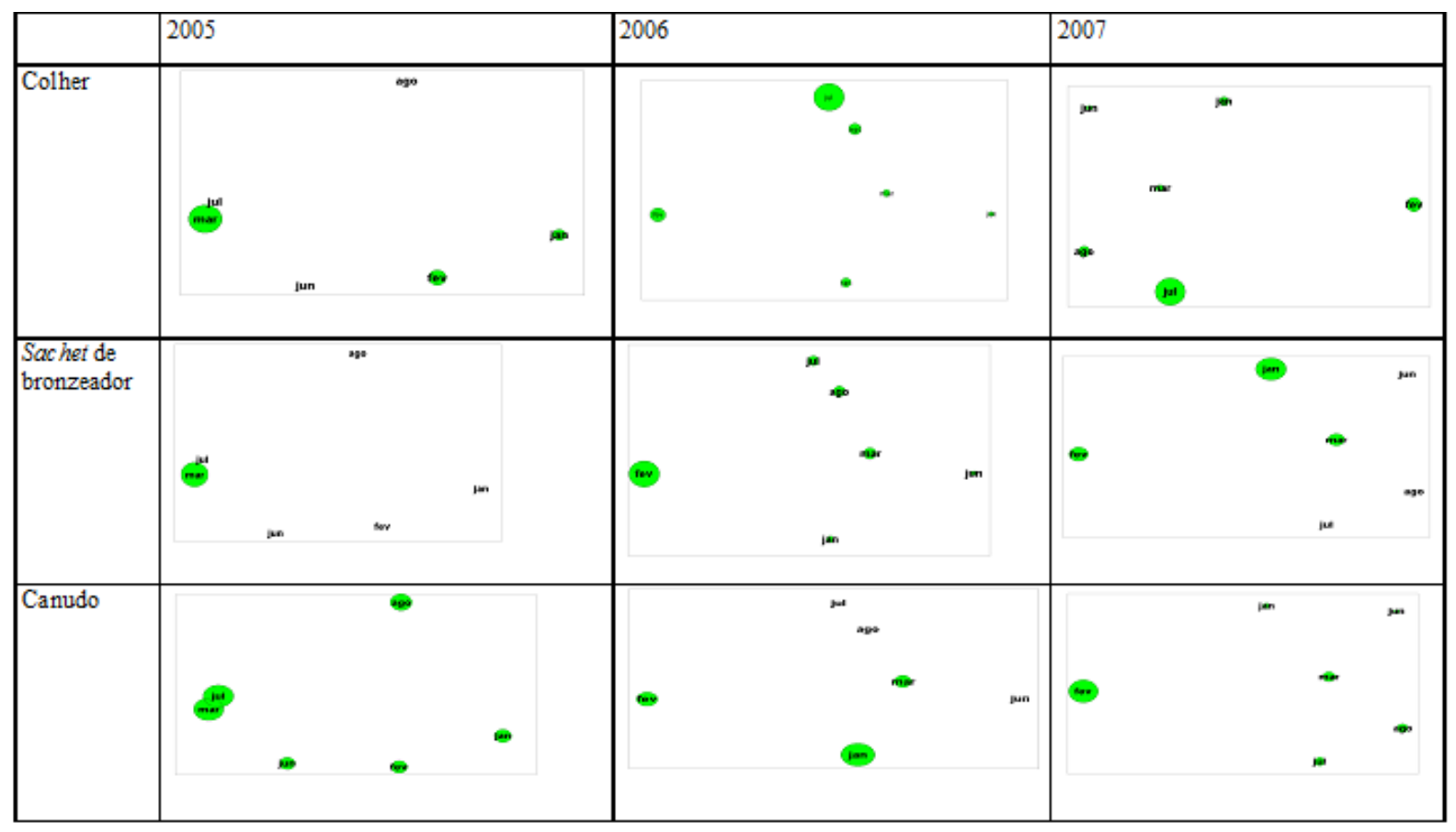

Figura 4: Ocorrência sazonal dos itens relacionados aos usuários da praia. Multi Dimensional Scaling (MDS) referente aos diferentes anos de coleta de resíduos sólidos na praia de Boa Viagem (stress: 0,01; 0; 0,01; 0,01; 0,01;0; 0,01; 0,01 e 0,01)

\section{Discussão}

Este trabalho confirma os resultados encontrados em outras pesquisas sobre a mesma temática em diversas outras partes do mundo (Morishige et al. 2007, Araújo \& Costa 2007, Araújo \& Costa 2006a, Araújo \& Costa 2004, Silva et al. 2009, Portz et al. 2011, Tourinho \& Filmann 2011, Vieira et al. 2011). Nelas, a categoria plásticos é a mais abundante entre os resíduos sólidos encontrados em praias. Isto demonstra a constância na proporção de plásticos na praia e no ambiente marinho adjacente, já relatada por diversos autores na literatura. Nossos resultados, como os de outros trabalhos sobre contaminação por resíduos sólidos de praias (Morishige et al. 2007, Araújo \& Costa 2006b, Araújo \& Costa 2007, Portz et al. 2011, Tourinho \& Filmann 2011, Vieira et al. 2011), refletem o aumento do uso de plástico nas últimas décadas, bem como suas características de durabilidade e alta flutuabilidade, que permitem sua permanência no mar durante tempo indeterminado.

A descoberta da fonte dos resíduos é fundamental para agir efetivamente sobre o problema da contaminação nas áreas costeiras. Os usuários, o aporte do rio Jaboatão e a atividade de pesca foram as principais fontes identificadas para a praia de Boa Viagem. Foi comprovado que a principal fonte de resíduos sólidos que contaminam a praia está relacionada ao seu usuário (80\%).

Assim, a intervenção humana direta é o principal responsável pela contaminação de praias recreacionais. De acordo com Silva et al. (2008), existe um padrão de distribuição espaço-temporal dos usuários na praia de Boa Viagem, e a distribuição de resíduos sólidos relacionados aos usuários segue o mesmo padrão
(Silva-Cavalcanti et al. 2009). Józwiak (2005) assume que a quantidade de lixo recreacional ao longo da linha de costa aumenta com o aumento dos usuários da praia. Ele observou a existência de correlação entre a presença de embalagem de comida e a quantidade de usuário na costa da Polônia. A quantidade média de embalagens de comida encontrada em dez anos de amostragem foi de 18 itens $/ \mathrm{km}$. Para a praia de Boa Viagem a quantidade de embalagens foi mais de cinco vezes maior (98 itens $/ \mathrm{km}$ ) do que a relatada por Józwiak (2005).

A relação entre a quantidade de usuários e a quantidade de resíduos sólidos na linha do deixa da praia de Boa Viagem foi bem estudada por SilvaCavalcanti et al. (2009) e Dias-Filho et al. (2011b); em ambos os trabalhos, foi observado que canudinhos e copos aumentam proporcionalmente com o número de usuários. No presente trabalho, foi observado que alguns itens relacionados aos usuários da praia (sachet de bronzeador, copos, entre outros) apresentaram certa sazonalidade em sua ocorrência, sendo muito maior durante a alta estação (dezembro a fevereiro), especialmente durante as férias escolares de verão (dezembro a janeiro) e inverno (julho). Ballance et al. (2000) apontam a limpeza da praia como um importante fator na escolha do usuário sobre qual praia visitar. Turistas e usuários afirmam não mais retornar à praia caso a quantidade de resíduos na areia seja maior do que 10 itens por metro linear (Ballance et al. 2000). Já, no presente estudo, o maior valor médio de itens encontrados por metro linear de praia foi de 5.811 Desvpad \pm 8.21 itens/m. Esses números são preocupantes por se encontrarem próximos dos valores apontados por Ballance et al. (2000) como críticos para os usuários. No entanto, é importante ressaltar que a 
percepção da qualidade da praia e sua tolerância, pelos usuários de Boa Viagem, pode se diferenciar em relação aos usuários da Península do Cabo.

Vários fatores oceanográficos e meteorológicos influenciam no transporte superficial de objetos no mar (Debrot et al. 1995). Embora Boa Viagem seja uma praia dissipativa, com pouca variação sazonal no volume sedimentar, ela possui trechos com cordões recifais próximos à linha d’água, que limitam a circulação de água. Ademais, sofre forte influência dos ventos, especialmente de SE, que atuam deslocando a pluma dos rios Jaboatão e Pirapama ao longo da praia, favorecendo o transporte de sedimentos e provavelmente também de resíduos.

Durante o período de estudo, foi constatado que os valores de velocidade do vento e de precipitação ficaram acima da média histórica, podendo refletir efeitos do El-Niño na região. Morishige et al. (2007) compararam a quantidade de resíduos sólidos em French Frigate Shoals (Havaí) durante os meses de ElNiño, La-Niña, e em períodos de ausência desses dois eventos. Eles observaram que em meses de El-Niño a quantidade de plástico foi bem maior do que na ocorrência de outros eventos.

A diferença entre o número de itens encontrados no inverno e no verão pode ser atribuída a fatores como precipitação e velocidade do vento. A precipitação média de 2006 não foi significativamente diferente em relação à média histórica $(\mathrm{p}>0,05)$. No entanto, ela encontrou-se acima da referida média, bem como dos outros anos (2000-2007). Este fato pode explicar uma maior quantidade de resíduos sólidos no inverno de 2006. Apesar de o inverno de 2006 ter se caracterizado por uma maior quantidade de resíduos sólidos, a maior diversidade foi encontrada no verão e inverno de 2005. Os dados deste trabalho mostraram que essa diferença se deve provavelmente à influência do El-Niño no ano de 2005 (www.inpe.gov.br).

O índice de diversidade de plásticos no verão de 2005 foi significativamente maior do que nos verões subsequentes. A velocidade do vento durante o verão de 2005 situou-se acima da média histórica recente (2000 a 2007). A média histórica da velocidade do vento para os meses de janeiro, fevereiro e março foi de, respectivamente, 2,4 Desvpad \pm 0,3; 2,3 Desvpad \pm 0,3 e 2,4 Desvpad $\pm 0,4 \mathrm{~m} . \mathrm{s}^{-1}$. Para o ano de 2005, os valores da velocidade do vento encontrados foram 2,7 , 2,5 e 2,4 m.s ${ }^{-1}$ para janeiro, fevereiro e março, respectivamente. Saliente-se que o aumento na velocidade do vento observado no verão de 2005 pode ter influenciado a deposição dos resíduos sólidos em geral e especialmente os plásticos, por sua alta flutuabilidade. A velocidade do vento pode ter influenciado também na direção da corrente de forma a permitir que a pluma do rio Jaboatão chegasse até a praia de Boa Viagem (Rollnic et al. 2004, Rollnic 2008), aumentando assim a diversidade de resíduos sólidos no verão de 2005. Observando os boletins de balneabilidade da CPRH para os anos de 2005 e 2006, foi possível verificar que no mês de janeiro, quando a velocidade média do vento registrada foi a maior do período amostrado $\left(2,7 \mathrm{~m} . \mathrm{s}^{-1}\right)$, a classificação da praia foi mais frequentemente imprópria, diferentemente do observado para o mesmo mês nos anos subsequentes (2006-2007) para os mesmos pontos de coleta (postos de salva-vidas 5 e 12) (Gondra et al. 2007). Nesses pontos a praia é aberta, sem a presença de arrecifes, ficando sob influência direta da pluma do rio Jaboatão (Araújo \& Costa 2008) e sem retenção nas piscinas naturais.

O fator hidrodinâmico tem um importante papel nos locais sob influência de rios. O estuário de Barra de Jangada (rios Jaboatão e Pirapama) está a 8 e 16 km dos limites sul e norte, respectivamente, da área amostral da praia de Boa Viagem. Sementes, folhas e troncos de Rhizophora mangle (mangue vermelho), espécie característica de áreas estuarinas, são comuns na praia de Boa Viagem. Vários aspectos neste trabalho corroboram a hipótese da chegada da pluma do rio Jaboatão até o limite norte da área estudada. Sendo o lixo de origem terrestre (aporte fluvial), a balneabilidade da praia e a presença de vegetação característica de estuário são os indícios mais fortes a corroborar esta hipótese.

O volume de água do rio Jaboatão e a velocidade e direção do vento para o período de monitoramento dos resíduos sólidos foram provavelmente os principais responsáveis por este aporte para a praia de Boa Viagem.

Durante o inverno, o aumento da precipitação ocasiona a chegada de maior volume de água doce na praia. Nessa época do ano a prevalência de ventos de Sudeste (SE) empurra a pluma do rio em direção à praia (Rollnic et al. 2004, Rollnic 2008). Desta maneira, os itens gerados em terra pelas comunidades adjacentes ao rio Jaboatão acabam sendo transportados pelo rio e depositados na praia. A chegada desses resíduos acaba por aumentar a diversidade dos resíduos sólidos, principalmente os plásticos, devido às suas características composicionais e comportamento ambiental.

Entre os itens mais abundantes relacionados aos usuários da praia estavam as bitucas de cigarros (filtros). Esses resíduos possuem um elevado potencial de acumulação na praia, porque além de serem descartados em grande quantidade, seu tamanho reduzido e coloração clara fazem com que se misturem e fiquem camuflados na areia, sendo pouco recolhidos pelos serviços de limpeza. A produção e soterramento desses resíduos durante a época de maior uso da praia podem se constituir num reservatório, que pode ser remobilizado pela dinâmica costeira e aumentar a quantidade de resíduos presentes, mesmo em épocas de menor uso.

\section{Conclusões}

A qualidade de vida da população brasileira melhorou nos últimos anos. Com o aumento da qualidade de vida, aumentou também o padrão de consumo em geral, bem como o investimento em lazer. 
Por ser a praia de Boa Viagem uma praia altamente acessível aos moradores da Região Metropolitana do Recife, torna-se destino certo entre as opções de lazer para o final de semana. O aumento da qualidade de vida da população ao longo dos anos ocasionou um aumento do número de usuários da praia, aumento do comércio informal para atender as necessidades básicas destes usuários e, consequentemente, um aumento da contaminação por resíduos sólidos.

A diversidade e deposição de resíduos sólidos na praia de Boa Viagem estão diretamente relacionadas a duas principais fontes: seus usuários e o aporte do rio Jaboatão. Durante o verão, existe a tendência de acumulação de resíduos sólidos relacionados ao usuário da praia. No inverno, além dos resíduos dos usuários, o aporte do rio é responsável pela maior quantidade e diversidade de lixo. O primeiro ano de ElNiño (2005) foi completamente atípico se comparado aos posteriores com relação à diversidade de resíduos sólidos encontrados na linha do deixa da praia de Boa Viagem. A precipitação e a velocidade do vento foram os principais fatores responsáveis pela diversidade e quantidade de resíduos sólidos encontrados. O padrão da diversidade de resíduos na praia de Boa Viagem mostrou um aumento durante a estação chuvosa, onde o aporte do rio e o regime de chuva e ventos são os responsáveis pela chegada e deposição de resíduos na praia. A quantidade de resíduos sólidos aumentou ao longo dos anos na praia de Boa Viagem. Estes resultados são preocupantes quando se considera a importância desta praia como cartão postal da cidade e no contexto econômico para a população local.

Trabalhos de educação ambiental podem ser realizados com os banhistas e vendedores de praia a fim de explicar os conceitos básicos de economia ambiental, destacando os aspectos de conservação relacionados diretamente à renda sustentável. A intensificação dos serviços de limpeza e a ampliação do número de lixeiras também são ações necessárias para uma melhor gestão da praia.

\section{Referências}

Araújo M.C.B., Costa M.F.C. 2008. Environmental quality indicators for recreational beaches classification. Journal of Costal Research, 24 (6):1439-1949.

Araújo M.C.B., Costa M.F.C. 2007. An analysis of the riverine contribution to the solid waste contamination of an isolated beach at the Brazilian Northeast. Management of Environmental Quality, 18(1):6-12.

Araújo M.C.B., Costa M.F.C. 2006a. Ideal width transects for monitoring source-related categories of plastics on beaches. Marine Pollution Bulletin, 52: 957-961.

Araújo M.C.B., Costa M.F.C. 2006b. Municipal services on Tourist beaches: cost and benefits of solid waste collection. Journal of Coastal Research, 22(5):1070-1075.

Araújo M.C.B., Costa M.F.C. 2004. Quali-quantitative analysis of the solid waste at Tamandaré Bay, Pernambuco, Brazil. Tropical Oceanography, 32(2):159-170.

Ballance A., Ryan P.G., Turpie J.K. 2000. How much is a clean beach worth? The impact of litter on beach users in the Cape Peninsula, South Africa. South African Journal of Science, 96:210-213.
Debrot O.A., Bradshaw J.E., Tiel A.B. 1995. Tar contatmination in Curaçao, Netherlands Antilles. Marine Pollution Bulletin, 30:689-693.

Dias-Filho M.J.O., Silva-Cavalcanti J.S., Araujo M.C.B., Silva A.C.M. 2011a. Avaliação da Percepção Pública na Contaminação por Lixo Marinho de acordo com o Perfil do Usuário: Estudo de Caso em uma Praia Urbana no Nordeste do Brasil Revista da Gestão Costeira Integrada 11(1):49-55 / Journal of Integrated Coastal Zone Management, 11(1):49-55.

Dias-Filho M.J.O., Araujo M.C.B., Silva-Cavalcanti J.S., Silva A.C.M. 2011b. Contaminação da praia de Boa Viagem (Pernambuco-Brasil) por lixo marinho: relação com o uso da praia. Arq. Ciên. Mar, Fortaleza, 44 (1):36-39.

Galgani F., Leaute J.P., Moguedet P., Souplets A., Verin Y., Carpentier A., Goraguer H., Latrouite D., Andral B., Cadiou Y., Mahe J.C., Poulard J.C., Nerisson P. 2000. Litter on the sea floor along Europeans Coast. Marine Pollution Bulletin, 40 (6):516527.

Gondra A.P.X., Tinoco S.C.B., Costa M.F. 2007. Análise preliminar das condições de balneabilidade das praias do litoral de Pernambuco, Nordeste do Brasil, de abril de 2005 a novembro de 2006. In: XII COLACMAR, 2007, Florianópolis. XII COLACMAR, 2007. p. 1-4.

Gregório M. das N., Araújo T.C.M. 2003. Caracterização volumétrica das praias do Pina e da Boa Viagem, Recife (PE) Brasil. IX Congresso da Associação Brasileira de Estudos do Quaternário.Recife-PE.

Hess N.A., Ribic C.A., Vining I. 1999. Benthic marine debris, with an emphasis on fishery-related items, sorrounding Kodiak Island, Alaska, 1994-1996. Marine Pollution Bulletin, 38 (10): 885-890.

Józwiak T. 2005. Tendencies in the number of beverage containers on the Polish coast in the decade from 1992-2001. Marine Pollution Bulletin, 50:87-90.

Leite A.P. de A. 2009. Levantamento ambiental e pesqueiro dos rios Jaboatão e Pirapama no estado de Pernambuco, Brasil. Dissertação (Mestrado em Recursos Pesqueiros e Aquicultura) Universidade Federal Rural de Pernambuco. 67 p.

Morishige C., Donohue M.J., Flint E., Swenson C., Woolaway C. 2007. Factors affecting marine debris deposition at French Frigate Shoals, Northwestern Hawaiian Islands Marine National Monument, 1990-2006. Marine Pollution Bulletin, 54, (8):1621169.

Nóbrega A.S. de C. 2011. Fontes de contaminação no estuário do rio Capibaribe, Pernambuco. Monografia. Bacharelado em Ciências Biológicas. Universidade Federal de Pernambuco. 48p.

Portz L., Manzolli R.P., Ivar do Sul J.A. 2011. Marine debris on Rio Grande do Sul north coast, Brazil: spatial and temporal patterns. Journal of Integrated Coastal Zone Management, 11, (1):41-48.

Ribic C. 1998. Use of indicator items to monitor marine debris on a New Jersey beach from 1991 to 1996. Marine Pollution Bulletin, 36 (11):887-891.

Rollnic M., Medeiros C. 2004. Circulation of the Coastal Waters off Boa Viagem, Piedade and Candeias Beaches-PE, Brazil . In: 8th International Coastal Symposium, 2004, Itajaí, SC. Anais 8th International Coastal Symposium,. Itajaí : UNIVALI, 2004. $1: 40-40$.

Rollnic M. 2008. Dinâmica das ondas e circulação no litoral da região metropolitana do Recife: implicações para o transporte de sedimentos e estabilidade da costa. Tese de Doutorado. Universidade Federal de Pernambuco. 124.p

Santos I.R., Friedrich A.C., Barreto F.P. 2005. Overseas garbage pollution on beaches of Northeast Brazil. Marine Pollution Bulletim, 50(7):783-786.

Silva-Iñiguez L., Fischer D.W. 2003. Quantification and classification of marine litter on the municipal beach of Ensenada, Baja California, Mexico. Marine Pollution Bulletin, 46, 132-135.

Silva J.S., Barbosa S.C.T., Leal M.M.V., Lins A.R., Costa M.F. 2006. Ocupação da praia da Boa Viagem (Recife/PE) ao longo de dois dias de verão: um estudo preliminar. Pan-American Journal of Aquatic Sciences, 1(2), 91-97.

Silva J.S., Leal M.M.V., Araújo M.C.B., Barbosa S.C.T., Costa M.F. 2008. Spatial and temporal patterns of use of Boa Viagem beach, Northeast Brazil. Journal of Coastal Research, 24 (1):79-86. 
Silva-Cavalcanti J.S., Araújo M.C.B., Costa M.F. 2009. Plastic litter an urban beach- a case study in Brazil. Waste Management \& Research, 27(1):93-97.

Smith R.A. 1981. Beach resorts: a model of development evolution: implications for management of resources. Canadian Geographer, 24(1):5-12.

Soares A.M., Costa M.F., Silva J.S., Araújo M.C.B. 2007. Contaminação da linha do deixa da praia da Boa Viagem (Recife-PE) por resíduos sólidos no verão de 2005: uma nova metodologia de avaliação. In: XII COLACMAR, 2007, Florianopolis. XII COLACMAR, 2007. p. 1-4.

Tourinho P.S., Ivar do Sul J.A., Fillmann G. 2007. Long term assessment of marine debris at Cassino beach, Rio Grande do Sul, Brazil. In: Anais da XIX Semana de Oceanografia.

Tourinho P.S., Filmann G. 2011. Temporal trend of litter contamination at Cassino beach, Southern Brazil. Journal of Integrated Coastal Zone Management, 11(1):97-102..

Vieira B.P., Dias D., Hanazaki N. 2011. Homogeneidade de encalhe de resíduos sólidos em um manguezal da Ilha de Santa Catarina, Brasil. Journal of Integrated Coastal Zone Management, 11(1):21-30.

Walker T.R., Reid K., Arnould J.P.Y., Croxall J.P. 1997. Marine debris surveys at Bird Island, South Georgia 1990-1995. Marine Pollution Bulletin, 34(1):61-65. . ${ }^{\text {i }}$

Recebido 26 de setembro de 2013

Aceito 04 de novembro de 2013 